\title{
Perdas econômicas ocasionadas pelas enfermidades podais em vacas leiteiras confinadas em sistema free stall
}

\author{
[Economic losses caused by sequels of lameness in free-stall-housed dairy cows] \\ R.C. Souza ${ }^{1}$, P.M. Ferreira ${ }^{2}$, L.R. Molina ${ }^{2}$, A.U. Carvalho ${ }^{2}$, E.J. Facury Filho ${ }^{2}$ \\ ${ }^{1}$ Pontifícia Universidade Católica - Betim, MG \\ ${ }^{2}$ Escola de Veterinária - UFMG \\ Caixa Postal 567 \\ 30123-970 - Belo Horizonte, MG
}

\begin{abstract}
RESUMO
Avaliaram-se 55 casos clínicos de manqueira em um rebanho de 100 vacas em lactação confinadas em sistema de free stall, durante um ano. As afecções mais observadas foram abscessos de sola e talão, úlcera de sola e dermatite digital que representaram $87,3 \%(48 / 55)$ das ocorrências. O tratamento empregado mostrou-se satisfatório com recuperação de todos os animais tratados com 24,5 dias, em média, sem ocorrência de descarte. O custo com tratamento e redução na produção de leite foi de US\$95.80/vaca, o que representou US\$52.69 por vaca alojada/ano. Quando se computaram as perdas reprodutivas e com mastite, obtiveram-se US\$227.94 adicionais em vaca com problema de manqueira. O custo adicional anual total no rebanho decorrente de seqüelas de manqueira foi de US\$12,536.70, que representou US\$125.36 por vaca alojada/ano. O período de serviço e o número de serviços por concepção em vacas com problemas de manqueira e normais foram 266 e 200,5 dias e 4,3 e 3,3 serviços, respectivamente. As incidências de mastite e metrite na mesma ordem de citação anterior foram $60 \%$ e $25 \%$ e $29 \%$ e $12,5 \%$.
\end{abstract}

Palavras-chave: vaca leiteira, laminite, dermatite digital, manqueira

\begin{abstract}
Fifty-five clinical cases of lameness were evaluated in 100 lactating cows housed in a free-stall system during one year. The most observed affections were sole and heel abscesses, sole ulcers and digital dermatitis that accounted for $87.3 \%$ (48/55) of the occurrences. The treated cows showed satisfactory recovery (24.5 average days) without culling. The total individual cost including treatment and reduction of milk production was US\$95.80/cow or US\$52.69 per housed cow/year. When reproductive losses and mastitis were considered an additional cost of US\$227.94 per lameness cow was estimated. The total additional annual cost from sequels of lameness was US\$12.536.70 or US\$152.36 per housed cow/year. The number of days open and the number of services per conception in lameness and normal cows were, respectively, 266 and 200.5 days and 4.3 and 3.3 services. Mastitis and metritis incidences, regarding the same animals, were, respectively, $60 \%$ and $25 \%$ and $29 \%$ and $12.5 \%$.
\end{abstract}

Keywords: dairy cow, laminitis, digital dermatitis, lameness

Recebido em 3 de agosto de 2005

Aceito em 22 de agosto de 2006

*Autor para correspondência (corresponding author)

E-mail: ferreira@vet.ufmg.br 


\section{INTRODUÇÃO}

As alterações podais de origem infecciosa envolvem primariamente a pele digital dos bovinos, apresentando incidência e prevalências elevadas em animais estabulados, especialmente em condições precárias de higiene, umidade excessiva e acúmulo de matéria orgânica. Elas são causas importantes de claudicações, particularmente a dermatite digital, um problema emergente em rebanhos leiteiros por todo o mundo. (Greenough e Weaver, 1997; Souza, 2002).

A etiologia das laminites é complexa, e sua patogênese incerta, sendo considerada multifatorial e importante causa de claudicação em bovinos. As principais seqüelas de laminite são hemorragias de sola, talão e linha branca, alterações da cor (amarelamento) e resistência dos tecidos córneos, doença da linha branca, úlceras de sola, linha branca e pinça, sola dupla, fissuras da muralha e erosões de talão (Greenough e Weaver, 1997).

Para Shearer et al. (1996) e Stanek (1997), as principais perdas por manqueiras são relacionadas à baixa produção de leite, redução da performance reprodutiva, descarte precoce, descarte de leite por uso de medicamentos, manejo adicional dos animais com problemas e morte. Na Inglaterra, Kossaibati e Esslemont (1997) observaram que manqueira era a terceira maior causa de perdas econômicas em vacas leiteiras.

A incidência anual de claudicação foi 30\% (Shearer et al., 1999), e o custo de US\$ $58,266.00 /$ ano, relativo à manqueira em um rebanho de 346 vacas, foi US\$ 357.00/vaca com problema e de US\$168.00/vaca alojada.

Cook (2002) encontrou prevalência média de $23 \%$ para claudicação em vacas leiteiras e custo adicional de US\$12,162.00/100 vacas confinadas, e de US\$122.00/vaca/ano. Na Europa, estudos sobre os custos com manqueira em vacas leiteiras relataram variações de US\$175.00 a US\$372.40/vaca alojada/ano (Esselemont, 1990; Whitaker et al., 1983).

Segundo Ferreira et al. (2004), a incidência anual de laminites foi $122 \%$, com custo de US\$44.00/animal tratado e US\$50.05, considerando apenas os custos com tratamento das seqüelas de laminite.

Segundo Collick et al. (1989), o período de serviço aumentou em 14 dias, e a taxa de prenhez ao primeiro serviço foi $46 \%$ nas vacas com manqueira contra $56 \%$ em vacas normais. Os animais com manqueira necessitaram 2,14 serviços por concepção contra 1,72 do grupocontrole, e a taxa de descarte $16 \%$ contra $5 \%$ no controle. Sagüés e Mazzucchelli (1998) relataram um período de serviço de 250 dias em vacas claudicantes. Outros estudos também têm mostrado associação entre claudicação e perdas na função reprodutiva (Butler e Smith, 1989; Collick et al., 1989; Wohlt et al., 1994).

Vacas claudicantes apresentam perda da condição corporal em decorrência da menor ingestão de alimento (Hassal et al., 1993). Segundo Gabarino et al. (2004), vacas claudicantes têm 3,5 vezes mais chance de apresentar diminuição das funções ovarianas se comparadas com as vacas normais. Dor, desconforto e perda da condição corporal podem resultar em imunossupressão, com conseqüente elevação de problemas de saúde, como mastite e metrite e de problemas reprodutivos (Gabarino et al., 2004).

O objetivo deste trabalho foi avaliar os prejuízos econômicos ocasionados por enfermidades podais em vacas leiteiras criadas em sistema de free stall.

\section{MATERIAL E MÉTODOS}

Estudaram-se 55 casos clínicos de manqueira causada por seqüelas de laminites e dermatite digital em um rebanho com 100 vacas da raça Holandesa em lactação, durante um ano, diagnosticadas durante as visitas à propriedade que ocorreram, em média, duas vezes por semana. Os animais foram examinados para avaliação do escore de claudicação de acordo com Sprecher et al. (1997). Os que apresentavam escore de claudicação igual ou superior a dois (manqueira clínica) foram identificados em ficha clínica para lesões podais e conduzidos para o tronco de casqueamento para contenção, limpeza e exame minucioso dos cascos. 
Utilizaram-se animais entre três e nove anos de idade, entre a primeira e a quinta lactação e com produção média de $10.000 / \mathrm{kg}$ de leite por lactação. Os animais, confinados em sistema de free-stall, com pedilúvio contendo solução de formalina a 5\%, trocada a cada 400 passagens; intercalada por solução de sulfato de cobre a $5 \%$, trocada a cada 200 passagens. No dia da secagem, as vacas foram submetidas a casqueamento preventivo conforme Toussaint Raven (1995).

Estudaram-se o período de serviço, o número de serviços por concepção e as ocorrências de metrite e mastite. Os casos de metrite foram de metrite puerperal aguda, que ocorriam no início da lactação. $O$ estudo dos custos incluiu: alimentação, mão-de-obra, sêmen e manejo adicional, além de despesas com medicamentos. Para fins de cálculo, todos os animais tiveram o período de lactação ajustado para 305 dias. Nos custos dos tratamentos, computaram-se os tempos de 40 minutos nas intervenções cirúrgicas e 25 minutos nas revisões dos casos com base na hora técnica sugerida pelo Conselho Regional de Medicina Veterinária de Minas Gerais. Os custos foram convertidos em dólares americanos da época e são apresentados na Tab. 1.

Tabela 1. Custos de fatores usados para avaliação das perdas econômicas ocasionadas por manqueiras em vacas leiteiras confinadas em sistema de free stall no município de Esmeraldas, MG

\begin{tabular}{lcc}
\hline Fator & Custo em reais & Custo em dólares(R\$1 $=$ \\
& & $\mathrm{US} \$ 0,33)$ \\
\hline Litro de leite & $\mathrm{R} \$ 0,54$ & $\mathrm{US} \$ 0.18$ \\
Alimentação/vaca em & $\mathrm{R} \$ 8,05$ & $\mathrm{US} \$ 2.69$ \\
produção/dia & & $\mathrm{US} \$ 13.00$ \\
Sêmen(dose) & $\mathrm{R} \$ 39,00$ & $\mathrm{US} \$ 47.00$ \\
Tratamento de mastite & $\mathrm{R} \$ 147,30$ & $\mathrm{US} \$ 68.19$ \\
Tratamento de metrite & $\mathrm{R} \$ 204,00$ & $\mathrm{US} 1.34$ \\
Período de serviço/dia & $\mathrm{R} \$ 4,02$ & \\
\hline
\end{tabular}

O período e o número de serviços por concepção, mastite e metrite foram comparados usando-se o pacote estatístico do MINITAB, versão 14.1, modelo 2003. Foi considerado um grau de significância de $\mathrm{P}<0,05$. Para os demais resultados observados, utilizou-se análise descritiva simples.

\section{RESULTADOS E DISCUSSÃO}

A incidência anual de manqueiras no rebanho foi $55 \%$ (55/100), sendo os abscessos de sola e talão, a úlcera de sola e a dermatite digital as causas mais presentes, com $87,3 \%(48 / 55)$ dos casos. $\mathrm{Na}$ Inglaterra, Russel et al. (1982) relataram incidência de manqueira entre 5,5\% e $65 \%$. Souza (2002) observou que dermatite e seqüelas de laminite foram as causas mais freqüentes de manqueiras em vacas leiteiras.

Os tratamentos das lesões foram satisfatórios, pois todos os animais tratados apresentaram-se curados, em um período médio de 24,5 dias. Ferreira et al. (2004) encontraram 26,8 dias. Segundo Mgasa (1987), as seqüelas de laminite são, em sua maioria, irreversíveis, o que determina número elevado de descartes. Ao final deste experimento, não houve descarte, talvez pelo fato de os animais terem sido acompanhados duas vezes por semana $\mathrm{e}$ submetidos a tratamento imediato ao mancarem, sem que houvesse demora do início da lesão ao tratamento. Dessa forma, a possibilidade de complicações, com acometimento das estruturas mais profundas, que inviabilizaria a permanência dos animais dentro do sistema de produção, foi eliminada.

O custo englobando o tratamento e a redução da produção leiteira no rebanho de 100 vacas foi de US\$5,269.00, sendo US\$95.80 o do tratamento e US\$52.69 o custo anual por vaca alojada. Esses resultados são semelhantes aos observados por Ferreira et al. (2004), que encontraram US\$5,005.23 para um rebanho de 117 vacas, com US\$44.68 representando o custo por animal tratado com seqüela de laminite. Os valores relacionados aos custos de tratamento, redução da produção leiteira, número de intervenções e período de tratamento encontram-se na Tab. 2. 
Perdas econômicas ocasionadas pelas enfermidades...

Tabela 2. Custo dos tratamentos e perda de produção leiteira decorrente das manqueiras em vacas leiteiras confinadas em um sistema de free stall em Esmeraldas, MG

\begin{tabular}{lcc}
\hline Fator & Valor observado & Custo (US\$) \\
\hline Redução média na produção leiteira por caso & $9,3 \mathrm{~kg}$ leite/dia & 1.68 \\
Duração média dos casos até a recuperação clínica & 24,5 dias & 41.20 \\
Número médio de intervenções por caso & 2,8 intervenções & 54.60 \\
Custo médio de tratamento por lesão & $\mathrm{R} \$ 287,40$ & 95.80 \\
Custo adicional anual por animal alojado & $\mathrm{R} \$ 158,07$ & 52.69 \\
Custo total no rebanho de 100 vacas & $\mathrm{R} \$ 15.807,00$ & $5,269.00$ \\
\hline
\end{tabular}

Foram necessárias 2,8 intervenções por caso clínico tratado. Ferreira et al. (2004) necessitaram de 3,83 intervenções, a cada sete dias, no tratamento de úlcera de sola. O menor número de intervenções por caso tratado neste estudo talvez tenha sido devido ao fato de as avaliações terem ocorrido com maior freqüência.

A redução média na produção leiteira por caso de manqueira, com perda total de $227 \mathrm{~kg}$, foi semelhante à obtida por Hernandez et al. (2000), quando a ocorrência de manqueira se deu no início da lactação.
Nas cirurgias, computou-se o valor de US\$28.00, e nos curativos, de US\$14.00. Ferreira et al. (2004), em Minas Gerais, observaram valores de US\$26.00 e de US\$12.00 para cirurgia e curativo, respectivamente.

Os valores adicionais relacionados à incidência de mastite, metrite, período de serviço e número de serviços em vacas mancas e normais encontram-se na Tab. 3, e os custos adicionais de todos os itens citados, na Tab. 4.

Tabela 3. Índices de mastite e problemas reprodutivos em vacas leiteiras com manqueira e normais, confinadas em um sistema de free stall em Esmeraldas, MG

\begin{tabular}{cccccccc}
\hline \multicolumn{2}{c}{ Período de serviço } & \multicolumn{2}{c}{$\begin{array}{c}\text { Número de serviços } \\
\text { por concepção }\end{array}$} & \multicolumn{2}{c}{ Metrite } & \multicolumn{2}{c}{ Mastite } \\
\hline $\begin{array}{c}\text { Com } \\
\text { manqueira }\end{array}$ & Normais & $\begin{array}{c}\text { Com } \\
\text { manqueira }\end{array}$ & Normais & $\begin{array}{c}\text { Com } \\
\text { manqueira }\end{array}$ & Normais & $\begin{array}{c}\text { Com } \\
\text { manqueira }\end{array}$ & Normais \\
\hline $266 \mathrm{a}$ & $200.5 \mathrm{~b}$ & $4.6 \mathrm{a}$ & $3.3 \mathrm{~b}$ & $25 \% \mathrm{a}$ & $12.5 \% \mathrm{~b}$ & $60 \% \mathrm{a}$ & $29 \% \mathrm{~b}$ \\
\hline
\end{tabular}

Valores seguidos de letras diferentes na mesma linha, para cada índice avaliado, diferem entre si $(\mathrm{P}<0,05) . \mathrm{N}=55$ e 45 , respectivamente, para vacas com manqueira e normal.

Tabela 4. Custos adicionais de mastite e de problemas reprodutivos em vacas leiteiras com manqueira e normais, confinadas em um sistema de free stall em Esmeraldas, MG

\begin{tabular}{lcc} 
Característica & $\begin{array}{c}\text { Diferença entre vacas } \\
\text { problema e normal }\end{array}$ & $\begin{array}{c}\text { Custo adicional em dólares } \\
\text { (US\$)/ vaca problema }\end{array}$ \\
\hline Período de serviço & 65,5 dias & 88.09 \\
Número de serviços & 1,3 serviço/concepção & 16.90 \\
Mastite & $31 \%$ incidência & 17.09 \\
Metrite & $12,5 \%$ incidência & 10.06 \\
Custo adicional decorrente de & & US\$132.14 \\
perdas reprodutivas e mastite & & US\$227.94 \\
Custo adicional anual total & & \\
\hline
\end{tabular}

O custo adicional/vaca atribuído aos problemas reprodutivos e metrite acrescido do custo do tratamento e da redução da produção de leite, US\$95.80, representou US\$ 227.94 a mais por vaca. $O$ custo adicional anual total no rebanho de 100 vacas, considerando $55 \%$ de incidência de manqueira, foi de US\$12,536.70, e o custo anual por vaca alojada de US\$125.36. Estes resultados são semelhantes aos encontrados por Cook (2002), que, ao estudar 30 rebanhos leiteiros nos Estados Unidos, formados por vacas com produção média de $8.600 \mathrm{~kg}$ por lactação, observou custo adicional total médio de US\$12,162.00 para 100 vacas, e de US\$122,00 
por vaca alojada/ano. Shearer et al. (1999), ao estudarem um rebanho leiteiro de 346 vacas na Flórida, computaram US\$168.00 por vaca alojada e Kossaibati e Esslemont (1997), na Inglaterra, US\$192.00.

Na Europa, diversos estudos sobre os custos de manqueira em vacas leiteiras relataram variações de US\$175.00 a US\$372.40 por vaca alojada no rebanho (Esselemont, 1990; Whitaker et al., 1983). Os custos observados neste experimento foram inferiores aos de diversos estudos realizados em outros países, provavelmente porque neles estão incluídos os custos adicionais decorrentes do aumento do descarte involuntário, o que não ocorreu neste trabalho.

O período de serviço das vacas claudicantes, 266 dias, foi semelhante ao relatado por Sagues e Mazzuccheli (1998), 250 dias, 65,5 dias a mais que em vacas normais $(\mathrm{P}<0,05)$. Estes resultados são próximos dos 77 dias encontrados por Hernandez et al. (2000). Alguns autores encontraram período de serviço mais longo em vacas acometidas (Colick et al., 1989; Lucey et al., 1986; Melendez et al., 2002).

As vacas claudicantes demandaram 1,3 serviços a mais(4,6 serviços/concepção) em relação às normais $(\mathrm{P}<0,05)$. Colick et al. (1989) observaram 2,14 serviços adicionais. O maior número de serviços por concepção e o aumento do período de serviço em vacas claudicantes, acarretando custo adicional ao sistema de produção, é também uma tendência observada por vários autores (Shearer et al., 1996; Stanek, 1997; Melendez et al., 2002; Sagues e Mazzucheli, 1998).

O escore da condição corporal dos animais com manqueiras foi 2,5, e dos normais, 3,0 $(\mathrm{P}<0,05)$. Hassal et al. (1993) observaram redução significativa da condição corporal em vacas que mancavam, enquanto Gabarino et al. (2004) relataram ser de 3,5 vezes maior a chance de apresentarem diminuição da função ovariana, caracterizada por cistos e redução na taxa de ovulação. Esses autores postularam que a diminuição da função ovariana poderia ser atribuída ao prolongamento do balanço energético negativo, em conseqüência de menor ingestão de alimentos no grupo de vacas com esse problema.

O aumento do período de serviço e do número de serviços por concepção pode ser atribuído ao fato de esses animais permanecerem menos tempo se alimentando. Essa redução seria conseqüência da alteração da função uterina em resposta à elevação dos níveis plasmáticos de ácidos graxos não esterificados e das cetonas, associada a baixas concentrações de glicose e insulina, atrasando o aumento nos pulsos de LH necessários para estimular o desenvolvimento dos folículos ovarianos, aspectos descritos por Goff e Horst (1997) e Butler (2000). A relação entre balanço energético e claudicação foi também uma tendência observada por Melendez et al. (2002). Esses autores verificaram perda de peso e menor ingestão de matéria seca em animais com manqueira. Postularam que a redução na ingestão de alimentos poderia afetar a síntese de hormônios esteróides, como a progesterona, mediante falta de precursores, o que interferiria no desenvolvimento inicial do embrião. A elevação dos níveis plasmáticos de $\mathrm{ACTH} /$ cortisol em vacas que claudicam pode inibir a síntese de GNRH ou de LH, o que interfere com as funções foliculares ovarianas (Butler e Smith, 1989; Collick et al., 1989; Lucey et al., 1986; Wohlt et al., 1994). A associação de fatores endócrinos e metabólicos, que resultaram em balanço energético negativo, com claudicação poderia explicar as perdas reprodutivas neste trabalho. Além disso, houve redução na demonstração de cio, conseqüentemente, diminuição da detecção de cio.

A incidência de mastite $(60 \%)$ e de metrite $(25 \%)$ em vacas claudicantes foi superior $(\mathrm{P}<0,05)$ à de vacas normais, respectivamente, $29,0 \%$ e $12,5 \%$, semelhante à descrita por outros autores (Shearer, 1996; Greenough e Weaver, 1997; Stanek, 1997; Shearer et al., 1999).

Segundo Melendez et al. (2002), vacas claudicantes experimentam dor e estresse que podem resultar em aumento dos níveis plasmáticos de catecolaminas, glicocorticóides, e essas substâncias são correlacionadas negativamente com doenças como mastite e metrite. Goff e Horst (1997) citaram que há associação entre fatores imunodepressores, como cortisol, e balanço energético negativo pelos corpos cetônicos, com imunossupressão e ocorrência de doenças secundárias. A redução na ingestão de alimentos em vacas com esse problema pode determinar deficiências de minerais e vitaminas essenciais para funcionamento normal do sistema imune. Assim, as ocorrências de mastite e metrite nas vacas claudicantes observadas neste trabalho podem ser atribuídas à imunossupressão resultante da elevação de cortisol e dos corpos cetônicos e pelas deficiências nutricionais. 


\section{REFERÊNCIAS BIBLIOGRÁFICAS}

BUTLER, W.R. Nutritional interactions with reproductive performanc in dairy catle. Anim. Reprod. Sci., v.60, p.449-457, 2000.

BUTLER, W.R.; SMITH, R.D. Interrelationships between energy balance and postpartum reproductive function in dairy cattle. J. Dairy Sci., v.72, p.767-783, 1989.

COLLICK, D.W.; WARD, W.R.; DOBSON, H. Associations between types of lameness and fertility. Vet. Rec., v.125, p.103-106, 1989.

COOK, N.B. Casco: doenças, custos, fatores de risco e prevenção. Novos enfoques na produção de bovinos. Conapec, Uberlândia, 2002. p.8-14.

ESSELEMONT, R.J. Costs of lameness, INTERNATIONAL CONFERENCE ON DISORDERS OF THE RUMINANT DIGIT, 6., 1990, Liverpool. Proceedings... Liverpool: University of Liverpool and British Cattle Veterinary Association, 1990. p.237-252.

FERREIRA, P.M.; LEITE, R.C.; CARVALHO, A.U. et al. Custos e resultados do tratamento das seqüelas de laminite bovina: relato de 112 casos em vacas em lactação no sistema de free-stall. Arq. Bras. Med. Vet. Zootec., v.56, p.589-594, 2004.

GABARINO, E.J.; HERNANDEZ, J.A.; SHEARER, J.K. et al. Effect of lameness on ovarian activity in postpartum holstein cows. J. Dairy Sci., v.87, p.41234131, 2004.

GOFF, J.P.; HORST, R.L. Physiological changes at parturition and their relationship to metabolic disorders. J. Dairy Sci., v.80, p.1260-1268, 1997.

GREENOUGH, P.R.; WEAVER, A.D. Lameness in cattle. 3.ed. Philadelphia: W.B.Saunders, 1997. 336p.

HASSAL, S.A.; WARD, W.R.; MURRAY, R.D. Effect of lameness on he behavior of cows during of summer. Vet. Rec., v.132, p.578-580, 1993.

HERNANDEZ, J.; SHEARER, J.K.; WEBB, D.W. Effect of papillomatous digital dermatitis and other lameness disorders on reproductive performance in a florida dairy herd. In: INTERNATIONAL SYMPOSIUM ON DISORDERS OF THE RUMINANT DIGIT, 11.; INTERNATIONAL CONFERENCE ON BOVINE LAMENESS, 3. Parma, Italia, 2000. p.300-302.

KOSSAIBATI, M.A.; ESSLEMONT, R.J. The costs of production diseases in dairy herds in England. Vet. J., v.154, p.41-51, 1997.
LUCEY, S.; ROWLANDS, G.J.; RUSSELL, AM. The association between lameness and fertility in dairy cows. Vet. Rec., v.118, p.628-631, 1986.

MELENDEZ, P.; BARTOLONE, J.; DONAVAN, A. Relationship betwen lameness, ovarian cysts and fertility in holstein cows. In: INTERNATIONAL SYMPOSIUM ON DISORDERS OF THE RUMINANT DIGIT, 12., Orlando, 2002. p.339-342.

MGASA, M.N. Bovine pododermatitis diffusa (laminitis), aetiology, pathogenesis, treatment and control. Vet. Res. Comm., v.12, p.235-241, 1987.

RUSSEL, A.M.; ROWLANDS, R.J.; WEAVER, A.D. Survey of lameness in British Dairy Cattle. Vet. Rec., v.21, p.155-160, 1982.

SAGÜÉS, G.A.; MAZZUCCHELLI, F. Control de cojeras en el Ganado vacuno de leche. Tratado de veterinária práctica bovis, Madrid: Luzáns, 1998. p.66-76.

SHEARER, J.K.; VAN AMSTEL, S.R.; MELENDEZ, P. Manual para el programa de recortador de pezuñas. Cojeras en el Ganado Lechero, marzo 4-6, 1999.

SHEARER, J.K.; BRAY, D.R.; UMPHREY, J.E. et al. Factors influencing the incidence of lameness in dairy cattle. In: ANNUAL FLORIDA DAIRY PRODUCTION CONFERENCE, 33., 1996. Gainesville: Proceedings... Gainesville: UF, 1996.

SOUZA, R.C. Perfil epidemiológico e clínico das afecções podais em vacas nas bacias leiteiras de Belo Horizonte e Pedro Leopoldo. 2002. 72f. Dissertação (Mestrado) - Escola de Veterinária, Universidade Federal de Minas Gerais, Belo Horizonte.

SPRECHER, D.J.; HOSTETLER, D.E.; KANEENE, J.B. A lameness scoring system that uses posture and gait to predict dairy cattle reproductive perfformance. Theriogenology, v.47, p.1179-1187, 1997.

STANEK, C. Housing and nutrition related claw diseases of dairy cattle. Israel J. Vet. Med., v.52, p.8087, 1997.

TOUSSAINT RAVEN, E. Cattle footcare and claw trimming. 5.ed. Ipswich: Farming, 1995. 127p.

WHITAKER, D.A.; KELLY, J.M.; SMITH, E.J. Incidence of lameness in dairy cows. Vet. Rec., v.113, p.60-62, 1983.

WOHLT, J.E.; ALLYN, M.E.; ZAJAC, P.K. et al. Cortisol increases in plasma of hostein heifer calves from handling and method of el ectrical dehorning. $J$. Dairy Sci., v.77, p.3725-3729, 1994. 